\title{
Evaluation of Anterior Segment Parameter Changes Using the Sirius after Uneventful Phacoemulsification
}

\author{
Ali Şimşek ${ }^{1}$, Burak Bilgin ${ }^{2}$, Musa Çapkın ${ }^{1}$, Şemsettin Bilak ${ }^{1}$, Mete Güler ${ }^{1}$, Ali Hakim Reyhan ${ }^{1}$ \\ ${ }^{I}$ Department of Ophthalmology, Adiyaman University School of Medicine, Adiyaman, Turkey \\ ${ }^{2}$ Department of Ophthalmology, Adiyaman Gozde Hospital, Adiyaman, Turkey
}

Purpose: To investigate changes in anterior chamber depth (ACD), corneal volume (CV), anterior chamber angle (ACA), anterior chamber volume (ACV), central corneal thickness (CCT), horizontal visible iris diameter (HVID), pupil diameter (PD), and intraocular pressure (IOP) after uneventful phacoemulsification cataract surgery with intraocular lens implantation.

Methods: A total of 132 eyes of 132 patients ( 87 men and 45 women) that underwent uneventful phacoemulsification cataract surgery and intraocular lens implantation were prospectively studied. The mean age of the patients was $63.68 \pm 12.51$ years. All patients were evaluated preoperatively and at 1 month postoperatively with the Sirius 3D Rotating Scheimpflug camera topography system. The ACD, CV, ACA, ACV, CCT, HVID, and PD measurements were recorded. IOP was measured using the Goldmann applanation tonometer, which was corrected for CCT of the Sirius device using Ehlers' formula.

Results: The preoperative mean ACD, ACV, ACA, CCT, CV, PD, HVID, and IOP were $2.79 \pm 0.45 \mathrm{~mm}, 124.73 \pm$ $25.72 \mathrm{~mm}^{3}, 42.09 \pm 7.49^{\circ}, 523.87 \pm 41.97$ microns, $55.37 \pm 4.89 \mathrm{~mm}^{3}, 3.98 \pm 1.23 \mathrm{~mm}, 11.72 \pm 0.67 \mathrm{~mm}$, and $14.74 \pm 2.59 \mathrm{mmHg}$, respectively. Three months postoperatively, the mean ACD, ACV, ACA, CCT, CV, PD, HVID, and IOP were $3.45 \pm 0.6 \mathrm{~mm}, 162.52 \pm 23.79 \mathrm{~mm}^{3}, 51.46 \pm 5.63^{\circ}, 526.21 \pm 44.45$ microns, $56.23 \pm 5.12$ $\mathrm{mm}^{3}, 2.87 \pm 0.45 \mathrm{~mm}, 11.91 \pm 0.75 \mathrm{~mm}$, and $12.02 \pm 1.83 \mathrm{mmHg}$, respectively. There was a statistically significant increase in mean postoperative ACD, ACV, ACA, CV, and HVID compared with the corresponding preoperative values $(p<0.05)$. CCT remained stable after surgery. Postoperative PD and IOP were significantly decreased compared to corresponding preoperative values $(p<0.05)$.

Conclusions: Preoperative measurements by the Sirius 3D Rotating Scheimpflug camera topography system might help surgeons to predict postoperative changes resulting from phacoemulsification and intraocular lens implantation. This is a noncontact, noninvasive, and comfortable system for patients that is highly reliable and repeatable for anterior segment measurements.

Key Words: Anterior eye segment, Corneal topography, Phacoemulsification

Anterior segment morphometry and intraocular pressure (IOP) can change after intraocular surgery. There are vari-

Received: June 15, 2015 Accepted: October 12, 2015

Corresponding Author: Burak Bilgin, MD. Department of Ophthalmology, Adıyaman Gozde Hospital, Eskisaray mah. 02040, Adiyaman, Turkey. Tel: 90-533-5601355, Fax: 90-553-4448202, E-mail: burbilgin@yahoo.com ous studies reporting these changes using different devices after phacoemulsification and intraocular lens (IOL) implantation [1-4]. Examining the anterior segment parameters is an important step for planning and evaluating the success of cataract surgery. Many clinical studies have reported that cataract extraction causes deepening of the anterior chamber, widening of the anterior chamber angle 
(ACA), and a significant decrease in IOP [5-11]. Traditional methods examining the anterior segment are not objective and do not provide quantitative information. Quantification of these changes has been assessed with different devices and methods. These new devices and methods are noncontact, noninvasive, and comfortable for patients in addition to providing highly reliable and repeatable anterior segment measurements; options include the Pentacam (Oculus Inc., Wetzlar, Germany) [1-4], anterior segment optical coherence tomography [12,13], and the Sirius system (Costruzione Strumenti Oftalmici, Florence, Italy) [3$15]$.

In this prospective study, we investigated changes in anterior chamber depth (ACD), corneal volume (CV), ACA, anterior chamber volume (ACV), central corneal thickness (CCT), horizontal visible iris diameter (HVID), pupil diameter (PD), and IOP after uneventful phacoemulsification cataract surgery and IOL implantation.

\section{Materials and Methods}

A total of 132 eyes of 132 patients ( 86 men and 46 women) that underwent uneventful phacoemulsification cataract surgery and IOL implantation between December 2013 and January 2014 were prospectively studied. The mean age of the patients was $63.68 \pm 12.51$ years. Exclusion criteria included history of trauma, ocular surgery, corneal pathology, pseudoexfoliation, uveitis, glaucoma, angle-closure, posterior segment pathology, diabetes, or use of topical or systemic medications that might affect anterior segment and IOP measurements. This study was approved by the Adiyaman University ethics committee, and the study protocol adhered to the tenets of the Declaration of Helsinki. Informed consent was obtained from each patient prior to any procedures.

All patients were examined preoperatively and 1 and 3 months postoperatively with the Sirius 3D Rotating Scheimpflug camera topography ystem, which was used to measure ACD, CV, ACA, ACV, CCT, HVID, and PD. IOP was measured using the Goldmann applanation tonometer and corrected for CCT of the Sirius device using Ehlers' formula. The patient was seated with the head positioned with the help of a chinrest and forehead strap during Sirius measurements. All measurements were obtained under standard dim light conditions without dilatation by the same trained examiner. To minimize diurnal corneal hydration variations, all measurements were obtained between 2 p.m. and 5 p.m.

The Sirius obtains topographic and anterior segment measurements using Scheimpflug photography. Its working principle is based on the combination of two rotating Scheimpflug cameras and a Placido disc with 22 rings.

All surgeries were performed by the same experienced surgeon (AŞ) under topical anesthesia with proparacaine drops (Alcaine; Alcon, Istanbul, Turkey). A 2.8-mm clear corneal incision was made in the superior quadrant. A continuous curvilinear capsulorhexis approximately $5.5 \mathrm{~mm}$ in diameter was completed after viscoelastic injection (Viscoat; Alcon Surgical, Fort Worth, TX, USA). Cortical cleaving hydrodissection was performed using balanced salt solution (Alcon Laboratories, Hemel Hempstead, UK). The nucleus was emulsified using torsional phaco technology (Infiniti, Alcon Laboratories) with the stop and chop technique. After cleaning the residual cortex with irrigation and aspiration, a foldable, acrylic, posterior chamber IOL (AcrySof, SA60AT; Alcon) was implanted into the capsular bag. To achieve wound integrity, stromal hydration was performed. Topical antibiotic and steroid drops were used postoperatively in all eyes. Topical antibiotic drops were applied four times a day for 1 week. Topical steroid drops were applied four times each day for 1 week and then tapered over 3 weeks.

Statistical analysis was performed with SPSS ver. 15.0 (SPSS Inc., Chicago, IL, USA). The paired $t$-test was used to compare variables between the preoperative and postoperative periods. Independent samples $t$-test was used to compare variables with a normal distribution between men and women before and after surgery. The Mann-Whitney $U$-test was used to compare variables without a normal distribution between men and women before and after surgery. Pearson's correlation test was used to evaluate the relationships among variables. A $p$-value less than 0.05 was considered statistically significant.

\section{Results}

The mean age of the patients was $63.68 \pm 12.51$ years (range, 15 to 87 years). Of 132 patients, $86(65.2 \%)$ were men and $46(34.8 \%)$ were women (Table 1). The mean ACD measurement was $2.79 \pm 0.45 \mathrm{~mm}$ preoperatively, 
$3.43 \pm 0.38 \mathrm{~mm} 1$ month postoperatively, and $3.45 \pm 0.6$ mm 3 months postoperatively. The mean ACV measurement was $124.73 \pm 25.72 \mathrm{~mm}^{3}$ preoperatively, $162.17 \pm$ $24.41 \mathrm{~mm}^{3} 1$ month postoperatively, and $162.52 \pm 23.79$ $\mathrm{mm}^{3} 3$ months postoperatively. The mean ACA measurement was $42.09 \pm 7.49^{\circ}$ preoperatively, $51.18 \pm 5.74^{0} 1$ month postoperatively, and $51.46 \pm 5.63^{\circ} 3$ months postoperatively. Mean CCT measurement was $523.87 \pm 41.97$ microns preoperatively, $526.80 \pm 47.86$ microns 1 month postoperatively, and $526.21 \pm 44.45$ microns 3 months postoperatively. Mean central $10 \mathrm{~mm} \mathrm{CV}$ measurement was $55.37 \pm 4.89$ $\mathrm{mm}^{3}$ preoperatively, $56.55 \pm 5.40 \mathrm{~mm}^{3} 1$ month postoperatively, and $56.23 \pm 5.12 \mathrm{~mm}^{3} 3$ months postoperatively. Mean PD measurement was $3.98 \pm 1.23 \mathrm{~mm}$ preoperatively, $2.90 \pm 0.48 \mathrm{~mm} 1$ month postoperatively, and $2.87 \pm 0.45$ mm 3 months postoperatively. Mean HVID measurement was $11.72 \pm 0.67 \mathrm{~mm}$ preoperatively, $11.92 \pm 0.751$ month postoperatively, and $11.91 \pm 0.75 \mathrm{~mm} 3$ months postoperatively. Mean IOP measurement was $14.74 \pm 2.59 \mathrm{mmHg}$ preoperatively, $12.11 \pm 2.16 \mathrm{mmHg} 1$ month postoperatively, and $12.02 \pm 1.83 \mathrm{mmHg} 3$ months postoperatively (Table 2).

There was a statistically significant increase in mean
$\mathrm{ACD}, \mathrm{ACV}, \mathrm{ACA}, \mathrm{CV}$, and HVID at 1 and 3 months postoperatively compared with corresponding preoperative values. Postoperative PD and IOP were statistically significantly decreased compared with corresponding preoperative values. There was not a significant change in CCT at 1 or 3 months postoperatively compared to the preoperative values.

The differences in all characteristics between 3 months postoperatively and preoperatively in women and men were not statistically significant (Table 3 ).

There was a statistically significant $(p<0.05)$ negative correlation between the 3 -month postoperative IOP values and ACD values $(r=-0.204)$. In addition, there was a negative correlation between the 3-month postoperative IOP values and $\mathrm{ACV}$ values $(\mathrm{r}=-0.223)$. There was a positive correlation between the 3-month postoperative IOP values and $\mathrm{CV}$ values $(\mathrm{r}=0.210)$ and a positive correlation between the 3-month postoperative IOP values and CCT values $(r=0.175)$. There was no statistically significant correlation between the 1 - and 3-month postoperative IOP values and other anterior segment parameters $(p>0.05)$ (Table 4).

Table 1. Demographic characteristics of the patients

\begin{tabular}{|c|c|c|c|c|c|}
\hline & Case & $\%$ & Range & Mean & Standard deviation \\
\hline Men & 86 & 65.2 & $29-87$ & 64.34 & 11.90 \\
\hline Women & 46 & 34.8 & $15-83$ & 62.46 & 13.63 \\
\hline Total & 132 & 100 & $15-87$ & 63.68 & 12.51 \\
\hline
\end{tabular}

Table 2. Comparison of preoperative and postoperative anterior segment parameters

\begin{tabular}{lccccc}
\hline & Preoperative & 1-mon postoperative & $p$-value & 3-mon postoperative & $p$-value \\
\hline ACD $(\mathrm{mm})$ & $2.79 \pm 0.45$ & $3.43 \pm 0.38$ & 0.000 & $3.45 \pm 0.36$ & 0.000 \\
CV $\left(\mathrm{mm}^{3}\right)$ & $55.37 \pm 4.89$ & $56.55 \pm 5.40$ & 0.000 & $56.23 \pm 5.12$ & 0.001 \\
ACA & $42.09 \pm 7.49$ & $51.18 \pm 5.74$ & 0.000 & $51.46 \pm 5.63$ & 0.000 \\
ACV $\left(\mathrm{mm}^{3}\right)$ & $124.73 \pm 25.72$ & $162.17 \pm 24.41$ & 0.000 & $162.52 \pm 23.79$ & 0.000 \\
CCT (microns) & $523.87 \pm 41.97$ & $526.80 \pm 47.86$ & 0.221 & $526.21 \pm 44.45$ & 0.186 \\
IOP (mmHg) & $14.74 \pm 2.59$ & $12.11 \pm 2.16$ & 0.000 & $12.02 \pm 1.83$ & 0.000 \\
HVID (mm) & $11.72 \pm 0.67$ & $11.92 \pm 0.75$ & 0.002 & $11.91 \pm 0.75$ & 0.003 \\
PD $(\mathrm{mm})$ & $3.98 \pm 1.23$ & $2.90 \pm 0.48$ & 0.000 & $2.87 \pm 0.45$ & 0.000 \\
\hline
\end{tabular}

Values are presented as mean \pm standard deviation. Paired $t$-test was used to compare preoperative and postoperative measurements. $\mathrm{ACD}=$ anterior chamber depth; $\mathrm{CV}=$ corneal volume; $\mathrm{ACA}=$ anterior chamber angle; $\mathrm{ACV}=$ anterior chamber volume; $\mathrm{CCT}=\mathrm{central}$ corneal thickness; IOP = intraocular pressure; HVID = horizontal visible iris diameter; $\mathrm{PD}=$ pupil diameter. 
Table 3. Comparison of 3-month postoperative and preoperative differences in all characteristics between women and men

\begin{tabular}{lcccc}
\hline & Women & Men & $t$-test & $p$-value \\
\hline ACD & $0.60 \pm 0.44$ & $0.70 \pm 0.38$ & 1.369 & 0.173 \\
ACA & $9.87 \pm 5.80$ & $9.093 \pm 5.19$ & 0.786 & 0.434 \\
ACV & $35.87 \pm 23.78$ & $38.80 \pm 28.11$ & 0.601 & 0.549 \\
PD & $-1.10 \pm 1.33$ & $-1.11 \pm 1.23$ & 0.062 & 0.951 \\
& Median & Median & U-test & 0.367 \\
CV & (first / third quarters) & (first / third quarters $)$ & $1,789.0$ & 0.175 \\
CCT & $0.40(-0.83 / 1.65)$ & $0.55(-0.33 / 2.05)$ & $1,694.5$ & 0.215 \\
IOP & $0.00(-6.00 / 3.00)$ & $1.00(-4.00 / 5.00)$ & $1,721.5$ & 0.189 \\
HVID & $-3.00(-4.25 /-1.00)$ & $-2.00(-4.00 /-1.00)$ & $1,703.0$ & $0.16(0.00 / 0.57)$ \\
Independ
\end{tabular}

Independent samples $t$-test was used for the variables with a normal distribution (ACD, ACA, ACV, and PD) and Mann-Whitney $U$-test was used for the variables that did not have a normal distribution (CV, CCT, IOP, and HVID).

$\mathrm{ACD}=$ anterior chamber depth; $\mathrm{ACA}=$ anterior chamber angle; $\mathrm{ACV}=$ anterior chamber volume; $\mathrm{PD}=$ pupil diameter; $\mathrm{CV}=\mathrm{corneal}$ volume; $\mathrm{CCT}=$ central corneal thickness; $\mathrm{IOP}=$ intraocular pressure; HVID = horizontal visible iris diameter.

\section{Discussion}

Pentacam (Oculus Optikgerate, Wetzlar, Germany) is the first device to use the Scheimpflug camera in ophthalmology practice. After Pentacam, Galilei (Ziemer Group, Port, Switzerland) introduced a combination of the Scheimpflug camera and Placido-disk topography system. Newer devices that use both the Scheimpflug camera and a Placido disk topography system are the Sirius (Costruzione Strumenti Oftalmici) and the TMS-5 (Tomey, Nagoya, Japan).

The Sirius topography device analyzes the anterior segment using a combination of a monochromatic three-dimensional (3D) rotating Scheimpflug camera and a Placido-disk with 22 rings. It obtains 25 radial sections of the cornea and anterior chamber. Scheimpflug cameras provide focused images from the anterior corneal surface to the posterior lens and transform them into a 3D model using their rotating capability [16]. Using a 3D rotating Scheimpflug camera, 21,632 points on the anterior corneal surface and 16,000 points on the posterior corneal surface are measured in less than 1 second. Anterior corneal surface measurement data are derived from both the Scheimpflug camera and Placido-disk images. Data for the posterior corneal surface and internal structures are derived only from the Scheimpflug camera.

In this study, we observed significant increase in ACD, $\mathrm{ACV}, \mathrm{ACA}, \mathrm{CV}$, and HVID and decrease in PD and IOP after uneventful phacoemulsification cataract surgery. Ac-
Table 4. Correlations between anterior segment parameters and intraocular pressure before operation and 1 and 3 months postoperatively $(\mathrm{n}=132)$

\begin{tabular}{llccc}
\hline & & \multicolumn{3}{c}{ Intraocular pressure } \\
\cline { 3 - 5 } & & Preoperative & $\begin{array}{c}1 \text {-mon } \\
\text { postoperative }\end{array}$ & $\begin{array}{c}\text { 3-mon } \\
\text { postoperative }\end{array}$ \\
\hline ACD & $\mathrm{r}^{*}$ & -0.015 & -0.254 & -0.204 \\
& $p^{\dagger}$ & 0.861 & 0.003 & 0.019 \\
$\mathrm{CV}$ & $\mathrm{r}$ & 0.213 & 0.211 & 0.210 \\
& $p$ & 0.014 & 0.015 & 0.016 \\
ACV & $\mathrm{r}$ & -0.062 & -0.188 & -0.223 \\
& $p$ & 0.478 & 0.031 & 0.010 \\
ACA & $\mathrm{r}$ & -0.062 & -0.175 & -0.136 \\
& $p$ & 0.482 & 0.045 & 0.121 \\
CCT & $\mathrm{r}$ & 0.145 & 0.255 & 0.175 \\
& $p$ & 0.096 & 0.003 & 0.044 \\
HVID & $\mathrm{r}$ & -0.069 & -0.138 & -0.156 \\
& $p$ & 0.430 & 0.114 & 0.074 \\
PD & $\mathrm{r}$ & 0.073 & 0.011 & 0.025 \\
& $p$ & 0.408 & 0.898 & 0.778 \\
\hline
\end{tabular}

Pearson correlation analysis was used to investigate the relationship between variables.

$\mathrm{ACD}=$ anterior chamber depth; $\mathrm{CV}=$ corneal volume; $\mathrm{ACV}=$ anterior chamber volume; $\mathrm{ACA}=$ anterior chamber angle; $\mathrm{CCT}=$ central corneal thickness; HVID = horizontal visible iris diameter; $\mathrm{PD}=$ pupil diameter.

"Pearson's correlation coefficient; ${ }^{\dagger} \mathrm{A} p$-value less than 0.05 was considered statistically significant. 
curate measurement of anterior chamber parameters is critical when planning surgery. In modern cataract surgery, to achieve desired refractive outcomes, correct IOL calculation with precise biometry is crucial. Accurately determining ACD is essential to achieve precise biometry. There are several methods to measure ACD [17,18]. Standard ultrasound devices define ACD as the distance between the anterior surface of the cornea and the anterior surface of the lens. Ultrasound devices are the most common method to measure ACD; however, as a contact device, ultrasound has some disadvantages such as corneal abrasions, infections, and off-axis measurement [18]. Sirius imaging uses Scheimpflug technology to measure ACD and is a relatively new and non-contact automatic optical technique.

There have been a number of studies using different devices, reporting marked anterior chamber changes following cataract surgery with phacoemulsification and foldable IOL implantation $[6,9,19,20]$. In our study, ACD and ACV increased 1.22- and 1.30-fold, respectively, compared to preoperative measurements. The results of our study are comparable with those of previous studies. Using Sirius, Takmaz et al. [3] reported a significant increase in ACD and ACV. The clinical meaning of these increases in ACD and $\mathrm{ACV}$ is more considerable for angle closure glaucoma patients who have a shallow anterior chamber.

Evaluation of ACA is critical in glaucoma patients. The conventional method used to evaluate ACA is gonioscopic examination. The major disadvantage of this method is subjectivity. ACA width can be quantitatively measured by Sirius. Using Sirius, Takmaz et al. [3] have reported a significant increase in ACA after uneventful phacoemulsification surgery and IOL implantation. Our study also showed a significant increase in ACA.

The effects of cataract surgery and IOL implantation on IOP have been shown in various studies. There are several studies in both normotensive eyes and glaucomatous eyes, including open angle glaucoma and angle closure glaucoma, reporting significant decreases in IOP after cataract surgery with phacoemulsification and foldable IOL implantation [8,19,21]. Altan et al. [9] reported that, in non-glaucomatous eyes with preoperative open ACA, uneventful phacoemulsification surgery reduced IOP, increased ACD, and widened the ACA.

The mechanism of the decrease in IOP resulting from phacoemulsification surgery is controversial. There are multiple possible mechanisms such as increased uveoscleral outflow, decreased resistance to conventional aqueous humour outflow, and hyposecretion of aqueous humour [22-25]. In our study, the mean IOP measured at 1 month postoperatively was significantly lower than mean preoperative value.

There are many recent methods to measure CCT, but ultrasound pachymetry is the older, gold standard method. Various studies comparing Pentacam measurements and ultrasound pachymetry have reported that Pentacam measurements are similar to but thinner than ultrasound pachymetry $[26,27]$. In our study, the mean CCT value was $523 \mu \mathrm{m}$ preoperatively. In a study by Doganay et al. [1] using Pentacam, the mean CCT was found to be $534 \mu \mathrm{m}$ preoperatively. Using Sirius, Takmaz et al. [3] found a mean CCT of $545 \mu \mathrm{m}$ preoperatively.

CCT is affected by corneal water content, which is maintained by endothelium pump and barrier functions. Corneal endothelial cell density and morphology can be assessed using specular microscopy in clinical practice, but the function of the endothelium pump, which is crucial for CCT and CV, cannot be assessed. Also, specular microscopic assessment can be performed in a limited corneal area. Therefore, it is difficult to statistically evaluate the entire cornea. The Sirius offers CV measurement in a central $10-\mathrm{mm}$ area of the cornea. The numerical value obtained can be used for statistical assessment.

To our knowledge, no previous study has compared CV changes before and after phacoemulsification surgery using The Sirius. Suzuki et al. [28] used Pentacam to evaluate $\mathrm{CV}$ changes in the central 3-mm and 10-mm areas after phacoemulsification surgery. They reported that the 3-mm area returned to preoperative values at 3 months after surgery, whereas the central 10 - $\mathrm{mm}$ area remained significantly higher than it had been preoperatively. In contrast to a previous study, Doganay et al. [1] reported no significant alterations in the 3-, 5- and 7-mm CV values after 1,3, or 6 months of uneventful phacoemulsification using Pentacam. In our study, we found a mean central 10-mm $\mathrm{CV}$ of $55.37 \mathrm{~mm}^{3}$ preoperatively, $56.55 \mathrm{~mm}^{3} 1$ month postoperatively, and $56.23 \mathrm{~mm}^{3} 3$ months postoperatively. There was a significant increase in $\mathrm{CV}$ after uneventful phacoemulsification surgery.

Pupil size is important for visual acuity and depends on the amount of light that enters the eye. This factor is particularly important in patients who have undergone 
phacoemulsification cataract surgery and multifocal IOL implantation. In their study using Pentacam, Doganay et al. [1] reported a mean preoperative pupil size of $2.6 \mathrm{~mm}$ and no significant change after surgery. In their study with Sirius, Takmaz et al. [3] reported a mean preoperative pupil size of $3.4 \mathrm{~mm}$; in accordance with a previous study, they did not find a significant difference between preoperative and postoperative values. In our study, mean PD was $3.98 \mathrm{~mm}$ preoperatively, $2.90 \mathrm{~mm} 1$ month postoperatively, and $2.87 \mathrm{~mm} 3$ months postoperatively. The mean postoperative PD was significantly lower than preoperative values.

In conclusion, after uneventful phacoemulsification cataract surgery and IOL implantation, significant increase in ACD , ACV, ACA, CV, and HVID and significant decrease in PD and IOP were recorded compared to preoperative measurements. There was not a significant change in CCT between preoperative and postoperative values. The Sirius allows for quick, easy, and reliable demonstration of anterior segment changes before and after phacoemulsification surgery and IOL implantation.

\section{Conflict of Interest}

No potential conflict of interest relevant to this article was reported.

\section{References}

1. Doganay S, Bozgul Firat P, Emre S, Yologlu S. Evaluation of anterior segment parameter changes using the Pentacam after uneventful phacoemulsification. Acta Ophthalmol 2010;88:601-6.

2. Cho YK, Chang HS, La TY, et al. Anterior segment parameters using Pentacam and prediction of corneal endothelial cell loss after cataract surgery. Korean J Ophthalmol 2010; 24:284-90.

3. Takmaz T, Kosekahya P, Kurkcuoglu PZ. Anterior segment morphometry and intraocular pressure change after uneventful phacoemulsification. Turk J Med Sci 2013;43:28993.

4. Dooley I, Charalampidou S, Malik A, et al. Changes in intraocular pressure and anterior segment morphometry after uneventful phacoemulsification cataract surgery. Eye
(Lond) 2010;24:519-26.

5. Issa SA, Pacheco J, Mahmood U, et al. A novel index for predicting intraocular pressure reduction following cataract surgery. Br J Ophthalmol 2005;89:543-6.

6. Kurimoto Y, Park M, Sakaue H, Kondo T. Changes in the anterior chamber configuration after small-incision cataract surgery with posterior chamber intraocular lens implantation. Am J Ophthalmol 1997;124:775-80.

7. Arai M, Ohzuno I, Zako M. Anterior chamber depth after posterior chamber intraocular lens implantation. Acta Ophthalmol (Copenh) 1994;72:694-7.

8. Cekic O, Batman C, Totan Y, et al. Changes in anterior chamber depth and intraocular pressure after phacoemulsification and posterior chamber intraocular lens implantation. Ophthalmic Surg Lasers 1998;29:639-42.

9. Altan C, Bayraktar S, Altan T, et al. Anterior chamber depth, iridocorneal angle width, and intraocular pressure changes after uneventful phacoemulsification in eyes without glaucoma and with open iridocorneal angles. J Cataract Refract Surg 2004;30:832-8.

10. Memarzadeh F, Tang M, Li Y, et al. Optical coherence tomography assessment of angle anatomy changes after cataract surgery. Am J Ophthalmol 2007;144:464-5.

11. Kashiwagi K, Kashiwagi F, Tsukahara S. Effects of small-incision phacoemulsification and intraocular lens implantation on anterior chamber depth and intraocular pressure. $J$ Glaucoma 2006;15:103-9.

12. Leung CK, Palmiero PM, Weinreb RN, et al. Comparisons of anterior segment biometry between Chinese and Caucasians using anterior segment optical coherence tomography. Br J Ophthalmol 2010;94:1184-9.

13. Kim M, Park KH, Kim TW, Kim DM. Changes in anterior chamber configuration after cataract surgery as measured by anterior segment optical coherence tomography. Korean J Ophthalmol 2011;25:77-83.

14. Jorge J, Rosado J, Diaz-Rey J, Gonzalez-Meijome J. Central corneal thickness and anterior chamber depth measurement by Sirius(R) Scheimpflug tomography and ultrasound. Clin Ophthalmol 2013;7:417-22.

15. Savini G, Barboni P, Carbonelli M, Hoffer KJ. Repeatability of automatic measurements by a new Scheimpflug camera combined with Placido topography. J Cataract Refract Surg 2011;37:1809-16.

16. Wegener A, Laser-Junga H. Photography of the anterior eye segment according to Scheimpflug's principle: options and limitations. A review. Clin Experiment Ophthalmol 
2009;37:144-54.

17. Nemeth G, Vajas A, Kolozsvari B, et al. Anterior chamber depth measurements in phakic and pseudophakic eyes: Pentacam versus ultrasound device. $J$ Cataract Refract Surg 2006;32:1331-5.

18. Koranyi G, Lydahl E, Norrby S, Taube M. Anterior chamber depth measurement: a-scan versus optical methods. $J$ Cataract Refract Surg 2002;28:243-7.

19. Hayashi K, Hayashi H, Nakao F, Hayashi F. Changes in anterior chamber angle width and depth after intraocular lens implantation in eyes with glaucoma. Ophthalmology 2000;107:698-703.

20. Pereira FA, Cronemberger S. Ultrasound biomicroscopic study of anterior segment changes after phacoemulsification and foldable intraocular lens implantation. Ophthalmology 2003;110:1799-806.

21. Nonaka A, Kondo T, Kikuchi M, et al. Angle widening and alteration of ciliary process configuration after cataract surgery for primary angle closure. Ophthalmology 2006; 113:437-41.

22. Miyake K, Asakura M, Kobayashi H. Effect of intraocular lens fixation on the blood-aqueous barrier. Am J Ophthal- mol 1984;98:451-5.

23. Alpar JJ. Glaucoma after intraocular lens implantation: survey and recommendations. Glaucoma 1985:7:241-5.

24. Meyer MA, Savitt ML, Kopitas E. The effect of phacoemulsification on aqueous outflow facility. Ophthalmology 1997;104:1221-7.

25. Kerstetter JR, Brubaker RF, Wilson SE, Kullerstrand LJ. Prostaglandin F2 alpha-1-isopropylester lowers intraocular pressure without decreasing aqueous humor flow. Am $J$ Ophthalmol 1988;105:30-4.

26. O'Donnell C, Maldonado-Codina C. Agreement and repeatability of central thickness measurement in normal corneas using ultrasound pachymetry and the OCULUS Pentacam. Cornea 2005;24:920-4.

27. Amano S, Honda N, Amano Y, et al. Comparison of central corneal thickness measurements by rotating Scheimpflug camera, ultrasonic pachymetry, and scanning-slit corneal topography. Ophthalmology 2006;113:937-41.

28. Suzuki H, Takahashi H, Hori J, et al. Phacoemulsification associated corneal damage evaluated by corneal volume. Am J Ophthalmol 2006;142:525-8. 